\title{
Nonmalignant hematology
}

\author{
Paul N. Knoebl (1)
}

Received: 10 July 2018 / Accepted: 17 July 2018 / Published online: 25 July 2018

(C) Springer-Verlag GmbH Austria, part of Springer Nature 2018

Esteemed reader,

MEMO is a journal primarily focusing on medical oncology and hematology, but it is also intended to "offer a professional review on current research and development in the field of hematology and oncology relevant for daily practice". Under that pretension, a broader view on aspects not directly associated with malignancy is encouraged and articles covering topics outside of that field have been and will be published in $M E M O$.

In this special issue on nonmalignant hematology four important topics are presented by distinguished experts: immune thrombocytopenia, hemoglobinopathies, and the two most important forms of thrombotic microangiopathies, thrombotic thrombocytopenic purpura and complement-mediated hemolytic uremic syndrome. All articles provide a comprehensive overview on pathophysiology, clinical picture, diagnostic methods and therapeutic options of these important syndromes.

Although these disorders are unique and frequently occur without an identifiable reason, there can be considerable overlap with malignant diseases: cancer itself, cancer medications, intercurrent infections, or transfusions can trigger immune responses, targeting platelets, ADAMTS13 or complement factors. Thrombotic microangiopathies are rare disorders, but are among the most challenging hematologic diseases. Their management requires skill, knowledge and clinical expertise. It is worth mentioning that thrombotic microangiopathies are sometimes associ-

Prof. P. N. Knoebl, MD (凶)

Department of Medicine 1, Div. Hematology and Hemostasis, Medical University of Vienna, Waehringer Guertel 18-20, 1090 Vienna, Austria

paul.knoebl@muv.ac.at ated with malignant diseases, having been triggered by malignancy or occurring as side effects of anticancer therapy, and special attention is required to untangle that network of interactions. This is also true for immune thrombocytopenia, an otherwise rather frequent disorder, but challenging when occurring in cancer patients. And even variants and abnormal forms of hemoglobin, which are common in some parts of Europe and are being seen more and more frequently in other locations due to increasing migration, can have an impact on the management of cancer patients. Thus, it is important to learn that not every abnormal blood lab or smear result suggests a malignant disorder but can also been caused by abnormal hemoglobin structure, or that certain transfusion triggers are not always useful in patients with variant hemoglobins.

The articles presented in this issue of $M E M O$ are intended to give an overview on the most important aspects of these disorders, but cannot cover all aspects in detail. We hope you enjoy reading and find the information useful for your daily work. Maybe you find some topics so interesting that you want to dive deeper into this field; our articles will guide you to advanced sources of information and experts in the field.

\section{Paul Knoebl}

Conflict of interest P.N. Knoebl declares that he has no competing interests.

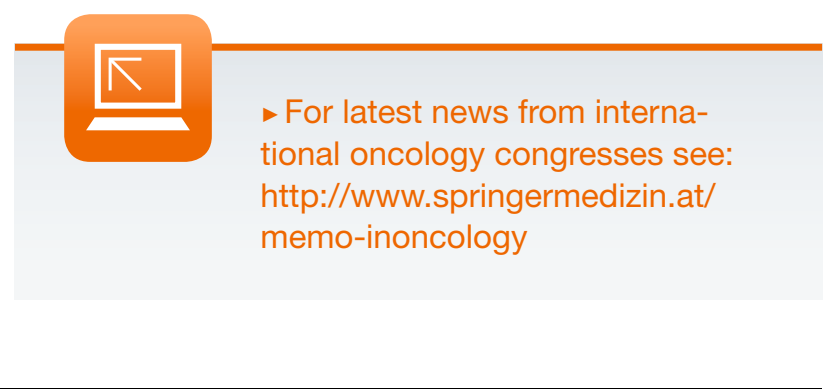

